\title{
Prognoses and Clinical Outcomes of Primary and Recurrent Uveal Melanoma
}

\section{Jee Hung Kim, $M D^{1,2}$ \\ Su-Jin Shin, MD, PhD \\ Soo Jin Heo, MD' \\ Eun-Ah Choe, MD, PhD' \\ Chang Gon Kim, MD1,4 \\ Minkyu Jung, MD, PhD ${ }^{1}$ \\ Ki Chang Keum, MD, PhD \\ Jin Sook Yoon, MD, $P h D^{6}$ \\ Sung Chul Lee, MD, $P h D^{6}$ \\ Sang Joon Shin, MD, $\mathrm{PhD}{ }^{1}$}

\begin{abstract}
${ }^{1}$ Division of Medical Oncology, Department of Internal Medicine, Yonsei Cancer Center, Yonsei University College of Medicine, Seoul, ${ }^{2}$ College of Medicine, Yonsei University Graduate School, Seoul, ${ }^{3}$ Department of Pathology, Hanyang University College of Medicine, Seoul, ${ }^{4}$ Graduate School of Medical Science and Engineering, KAIST, Daejeon, ${ }^{5}$ Department of Radiation Oncology, Yonsei Cancer Center, Yonsei University College of Medicine, Seoul, ${ }^{6}$ Institute of Vision Research, Department of Ophthalmology, Yonsei University College of Medicine, Seoul, Korea
\end{abstract}

\section{Purpose}

Uveal melanoma has a very poor prognosis despite successful local primary tumor treatment. In this study, we investigated prognostic factors that more accurately reflected the likelihood of recurrence and survival and delineated a prognostic model that could effectively identify different risk groups based on initial clinical parameters.

\section{Materials and Methods}

Prognostic factors associated with distant recurrence, recurrence-free survival (RFS), progression-free survival, and overall survival from distant recurrence to death (OS2) were analyzed in 226 patients with stage I-III uveal melanoma who underwent primary local therapy.

\section{Results}

Forty-nine patients (21.7\%) had distant recurrences, which occurred most frequently in the liver (87.7\%). In a multivariate analysis, local radiotherapy improved RFS among patients with multiple recurrence risk factors relative to excision (not reached vs. 19.0 months, $\mathrm{p}=0.004$ ). Patients with BRCA1-associated protein-1 (BAP1)-negative primary tumors showed a longer RFS duration after primary treatments, while those with BAP1-negative metastatic tissues had a shorter OS2 compared to those with BAP1-positive tumors, both not statistically insignificance (RFS: not reached vs. 82.0 months, $p=0.258$; 0S2: 15.7 vs. 24.4 months, $p=0.216$ ). Male sex (hazard ratio [HR], 3.79; $p=0.012$ ), a short RFS (HR, 4.89; $p=0.014)$, and a largest metastatic tumor linear diameter $\geq 45 \mathrm{~mm}(H R, 5.48 ; p=0.017)$ were found to correlate with worse post-recurrence survival.

\section{Conclusion}

Risk factors could be used to classify uveal melanoma cases and subsequently direct individual treatment strategies. Furthermore, metastasectomy appears to contribute to improved survival outcomes.

Correspondence: Sang Joon Shin, MD, PhD

Division of Medical Oncology,

Department of Internal Medicine,

Yonsei Cancer Center, Yonsei University

College of Medicine, 50-1 Yonsei-ro,

Seodaemun-gu, Seoul 03722, Korea

Tel: 82-2-2228-4320

Fax: 82-2-393-3652

E-mail: SSJ338@yuhs.ac

Received November 8, 2017

Accepted December 27, 2017

Published Online December 28, 2017

Key words

Uveal neoplasm, Melanoma, Recurrence, Survival, Prognosis, BAP1 


\section{Introduction}

Uveal melanoma, the most common primary intraocular cancer, has a very poor prognosis, with reported median overall survival durations of 4-15 months [1-3]. Uveal melanoma is usually asymptomatic and is often diagnosed incidentally during routine ophthalmic examinations. Accordingly, appropriate treatment is often delayed. Although primary uveal melanomas are often successfully eradicated, nearly $50 \%$ of patients develop systemic metastatic disease [2], which almost always involves the liver $(89 \%)$ [3]. Metastases of uveal melanoma are rarely detectable at the time of the first local ocular treatment, and recent studies have shown that patients undergo routine liver ultrasonography screening to detect metastatic sites [4]. However, no standard screening protocol for uveal metastases currently exists, and ultrasonography alone often fails to detect hepatic sites.

Chemotherapeutic agents such as dacarbazine or gemcitabine/treosulfan are often administered to patients in whom recurrent uveal melanoma has been detected, although limited evidence supports the use of these regimens. Specifically, these patients rarely exhibit clinical responses, and therefore the efficacy of systemic chemotherapy is questionable [1]. Currently, the immunotherapeutic agents nivolumab and pembrolizumab, which are fully human monoclonal antibodies specific for the programmed cell death protein 1 (PD-1) receptor, have been approved in the United States for the treatment of advanced melanoma. However, PD-1 inhibitors have not been demonstrated to improve the survival of patients with uveal melanoma, and previous studies reported a median progression-free survival (PFS) and overall response rate of only 3 months and $3.6 \%$, respectively $[5,6]$.

Several groups have studied the prognostic factors of uveal melanoma. For example, Harbour et al. [7] reported an inactivating somatic mutation of $B A P 1$, the gene encoding BRCA-associated protein 1 (BAP1), in predominantly metastatic uveal melanomas. This mutation was later found to correlate strongly with the risk of metastasis [8]. Another study demonstrated associations of ciliary body involvement, the largest tumor diameter, and extraocular extension with a significantly poor prognosis in patients with uveal melanoma [9].

In the present retrospective study, we identified prognostic predictors of distant recurrence and survival after recurrence in 226 patients with uveal melanoma who received primary local therapy at our hospital between 1990 and 2015. We aimed to study the time interval between the diagnoses of primary and recurrent uveal melanoma and the efficacies of systemic therapies after recurrence. We also delineated a prognostic model that could effectively identify different risk groups based on initial clinical parameters.

\section{Materials and Methods}

\section{Patients and data collection}

We retrospectively reviewed the medical records of 226 patients who were diagnosed with stage I-III uveal melanoma between January 1990 and December 2015 at the Department of Ophthalmology, Yonsei University College of Medicine and the Yonsei Cancer Center. The inclusion criteria were as follows: (1) a diagnosis of uveal melanoma, (2) treatment with local resection or radiotherapy at the time of diagnosis, (3) no distant metastases at the time of diagnosis, and (4) available clinical data at the time of treatment.

We collected baseline clinical variables, including age, sex, and co-morbidities, and tumor data, including location, size, initial stage, and histology. Histologic classification of the primary uveal melanoma was performed through the enucleation report, local excision or ciliary body excision. The largest basal diameter (LBD) and depth of each tumor were measured using B-scan ultrasonography (Ellex, Adelaide, SA, Australia). Tumor staging was based principally on the Guideline for Uveal Melanoma from the American Joint Committee on Cancer (AJCC) Cancer Staging Manual, seventh edition [10].

\section{Treatment}

Local radiotherapy was performed as described in previous studies [11,12]. Brachytherapy with ${ }^{106} \mathrm{Ru}$ plaques (Eckert \& Ziegler BEBIG, Berlin, Germany) was primarily performed as an eye-sparing treatment. The range of target radiation doses to the tumor apex was 85-100 Gy. Some patients also received adjuvant trans-pupillary thermotherapy (TTT) using a diode laser with a slit-lamp delivery system at 3-month intervals, based on therapeutic responses. The exposure duration was 1 minute per spot, and the laser power was adjusted until the tumor surface became grayto-white within 1 minute.

Primary enucleation was performed for large tumors. Patients who strongly refused enucleation underwent brachytherapy. A surgical biopsy was performed when histologic confirmation was required. External sclerouvectomy was generally performed for ciliary body melanoma and anterior choroidal melanoma; endoresection via trans pars plana vitrectomy (TPPV) was performed for posterior choroidal melanoma [13-15]. 
Table 1. Baseline characteristics of primary and metastatic uveal melanoma

\begin{tabular}{|c|c|c|c|c|}
\hline Characteristic & $\begin{array}{c}\text { Total } \\
(n=226)\end{array}$ & $\begin{array}{l}\text { No recurrence } \\
\quad(n=177)\end{array}$ & $\begin{array}{l}\text { Recurrence } \\
(n=49)\end{array}$ & p-value ${ }^{a}$ \\
\hline Age, median (range, yr) & $53.0(18-71)$ & $54.0(18-71)$ & $50.0(19-61)$ & 0.232 \\
\hline \multicolumn{5}{|l|}{ Sex } \\
\hline Male & $109(48.2)$ & $84(46.2)$ & $28(53.8)$ & 0.158 \\
\hline Female & $117(51.8)$ & $98(53.8)$ & $24(46.2)$ & \\
\hline \multicolumn{5}{|l|}{ Location } \\
\hline Choroid & $220(97.3)$ & $173(97.7)$ & $47(95.9)$ & 0.613 \\
\hline Ciliary body & $6(2.7)$ & $4(2.3)$ & $2(4.1)$ & \\
\hline \multicolumn{5}{|l|}{ Histology $(\mathrm{n}=99)$} \\
\hline Epithelioid & $43(43.4)$ & $33(53.2)$ & $10(27.0)$ & 0.024 \\
\hline Spindle & $18(18.2)$ & $11(17.7)$ & 7 (18.9) & \\
\hline Mixed & $38(38.4)$ & $18(29.0)$ & $20(54.1)$ & \\
\hline \multicolumn{5}{|l|}{ Size, median (range, mm) } \\
\hline Largest basal diameter & $10.2(1.10-30.9)$ & $10.0(1.1-21.0)$ & $12.0(4.0-32.0)$ & 0.001 \\
\hline Depth & $6.2(1.10-757.9)$ & $6.0(1.10-757.0)$ & $7.75(1.5-18.0)$ & 0.539 \\
\hline \multicolumn{5}{|l|}{ Stage $(n=104)$} \\
\hline I & $26(25.0)$ & $25(32.5)$ & $1(3.7)$ & 0.004 \\
\hline II & $66(63.5)$ & $45(58.4)$ & $21(77.8)$ & \\
\hline III & $12(11.5)$ & $7(9.1)$ & $5(18.5)$ & \\
\hline \multicolumn{5}{|l|}{ Local treatment } \\
\hline $\mathrm{RTx}^{\mathrm{b})}$ & $160(70.8)$ & $136(76.8)$ & $24(49.0)$ & $<0.001$ \\
\hline Excision $^{c)}$ & $66(29.2)$ & $41(23.2)$ & $25(51.0)$ & \\
\hline \multicolumn{5}{|l|}{ Co-morbidity } \\
\hline None & $139(61.5)$ & $107(60.5)$ & $32(65.3)$ & 0.772 \\
\hline Cardiovascular disease & $7(3.1)$ & $6(3.4)$ & $1(2.0)$ & \\
\hline HTN & $56(24.8)$ & $45(25.4)$ & $11(22.4)$ & \\
\hline $\mathrm{DM}$ & $22(9.7)$ & $18(10.2)$ & $4(8.2)$ & \\
\hline HBV carrier & $7(3.1)$ & $5(2.8)$ & $2(4.1)$ & \\
\hline Others $^{\mathrm{d})}$ & $17(7.5)$ & $14(7.9)$ & $3(6.1)$ & \\
\hline \multicolumn{5}{|l|}{ BAP1 expression (IHC) > 5\% } \\
\hline \multicolumn{5}{|l|}{ Primary tumor tissue $(n=69)$} \\
\hline No & $13(18.8)$ & $10(22.7)$ & $3(12.0)$ & 0.349 \\
\hline Yes & $56(81.2)$ & $34(77.3)$ & $22(88.0)$ & \\
\hline \multicolumn{5}{|l|}{ Metastasis tumor tissue $(\mathrm{n}=20)$} \\
\hline No & - & - & $7(35.0)$ & 0.354 \\
\hline Yes & - & - & $13(65.0)$ & \\
\hline \multicolumn{5}{|l|}{ Recurrence site } \\
\hline Liver & - & - & $43(87.7)$ & $<0.001$ \\
\hline Bone & - & - & $11(22.4)$ & \\
\hline Lung & - & - & $3(6.1)$ & \\
\hline Others ${ }^{\mathrm{e})}$ & - & - & $6(12.2)$ & \\
\hline \multicolumn{5}{|c|}{ First systemic treatment after recurrence } \\
\hline Chemotherapy $\left.{ }^{\mathrm{f}}\right)$ & - & - & $35(71.4)$ & - \\
\hline Immunotherapyg) & - & - & $4(8.2)$ & \\
\hline Radiotherapy ${ }^{\mathrm{h}}$ & - & - & $3(6.1)$ & \\
\hline TACE, RFA on liver metastasis & - & - & $4(8.2)$ & \\
\hline Metastatectomy $y^{\text {i) }}$ & - & - & $7(14.3)$ & \\
\hline Observation & - & - & $8(16.3)$ & \\
\hline
\end{tabular}

(Continued to the next page) 
Table 1. Continued

\begin{tabular}{|c|c|c|c|c|}
\hline Characteristic & $\begin{array}{c}\text { Total } \\
(n=226)\end{array}$ & $\begin{array}{l}\text { No recurrence } \\
\quad(n=177)\end{array}$ & $\begin{array}{l}\text { Recurrence } \\
\qquad(\mathrm{n}=49)\end{array}$ & p-value $\left.{ }^{a}\right)$ \\
\hline $\begin{array}{l}\text { Largest linear dimension of largest metastatic tumor }(\mathrm{n}=51) \text {, } \\
\text { median (range, } \mathrm{mm})\end{array}$ & - & - & $25.0(4-94)$ & - \\
\hline
\end{tabular}

Values are presented as number (\%) unless otherwise indicated. RTx, radiotherapy; HTN, hypertension; DM, diabetes mellitus; HBV, hepatitis B virus; BAP1, BRCA1-associated protein-1; IHC, immunohistochemistry; TACE, transarterial chemoembolization; RFA, radiofrequency ablation. ${ }^{\mathrm{a}}$ Comparison betTeen patients with experience of recurrence or not, ${ }^{\mathrm{b})}$ Includes brachytherapy, gamma-knife radiotherapy, and transpupillary thermotherapy, ${ }^{\circ}$ Includes enucleation, excision, exentration, and trans pars plana vitrectomy, ${ }^{\mathrm{d}}$ Includes benign prostate hyperplasia, old TBc, other malignancy, and thyroid disease, ${ }^{\mathrm{e}}$ Includes nasal cavity, breast, spleen, brain, and adrenal gland metastasis, fIIncludes dacarbazine, cisplatin, topotecan, dacarbazine+

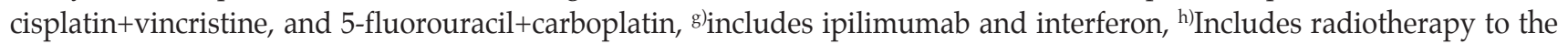
nasal cavity, spine, adrenal gland, and brain, ${ }^{\mathrm{i}}$ Includes splenectomy, liver wedge resection, adrenalectomy, lung lobectomy, skull craniectomy, and maxillectomy.

Forty-nine patients who developed distant recurrences following initial local treatment underwent metastasectomy and/or palliative first-line systemic treatments comprising chemotherapies and immunotherapies.

\section{Immunohistochemistry}

Tumor immunohistochemistry (IHC) was performed using a Ventana XT automated staining system (Ventana Medical Systems, Tucson, AZ) according to the manufacturer's protocol in the 69 primary and 20 metastatic uveal melanoma tissues. Four-micron-thick sections were immunostained with primary antibodies specific for BAP1 (1:50, C-4, mouse monoclonal, Santa Cruz Biotechnology, Dallas, TX), which yielded a nuclear staining pattern. BAP1 expression was determined according to the percentage of BAP1positive cells among all melanoma cells. Samples with a BAP1-positive cell frequency of $<5 \%$ were considered to have lost BAP1 expression.

\section{Statistical analysis}

For the analysis, datasets were classified as "before" or "after" distant recurrence. Before recurrence, we reviewed the primary tumor characteristics and local treatment efficacies. Recurrence-free survival (RFS) was defined as the time from the initial diagnosis of uveal melanoma to the time of distant recurrence. After recurrence, we reviewed medical records concerning recurrence patterns and the efficacies of systemic palliative treatment and surgery. The disease control rate (DCR) was defined as the proportion of patients who achieved a complete response, confirmed partial response, or stable disease per the Response Evaluation Criteria in
Solid Tumors (RECIST) ver. 1.1. PFS was defined as the time interval from the initiation of first-line systemic therapies to the date of documented disease progression or death from any cause. OS1 was measured from the date of initial diagnosis with uveal melanoma to the date of death from any cause, and OS2 was measured from the date of distant recurrence to the date of death from any cause.

The primary endpoint of the study, OS2, was calculated using the Kaplan-Meier product-limit method, and survival rates were compared statistically using the log-rank test and generalized Wilcoxon test. All univariate analyses included the following factors: age, sex, histology, size, tumor stage and location, distant metastasis pattern, and treatment modality. The multivariate analysis was performed using stepwise Cox proportional hazards regression modeling. $\mathrm{p}$-values of $<0.05$ were considered statistically significant, and all p-values corresponded to two-sided significance tests. Data were analyzed using SPSS software ver. 23.0 (IBM Corp., Armonk, NY).

\section{Ethical statement}

This study was reviewed and approved by the Institutional Review Board of Yonsei Cancer Center (IRB 4-20160300). Our institutional review board approved this retrospective study and waived the requirement to obtain informed consent. 
A

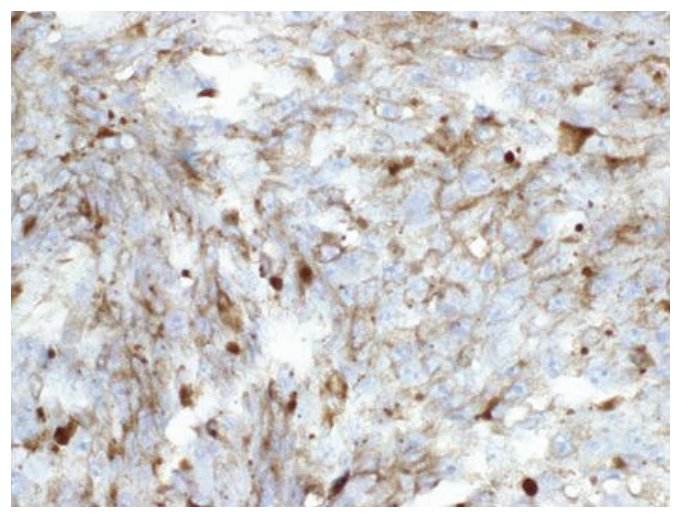

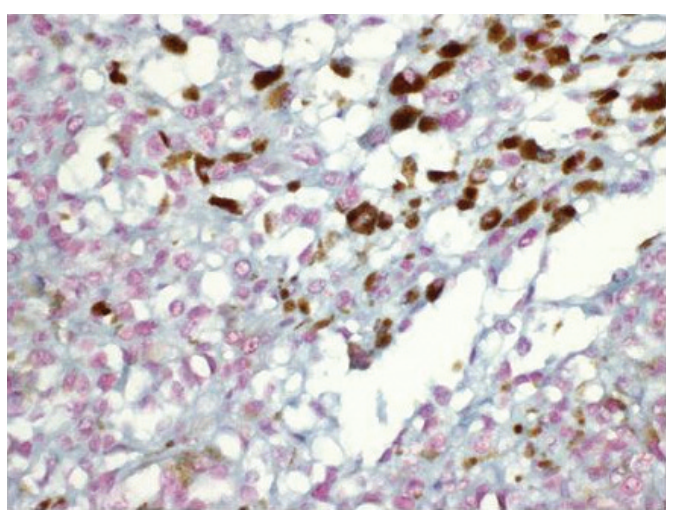

D

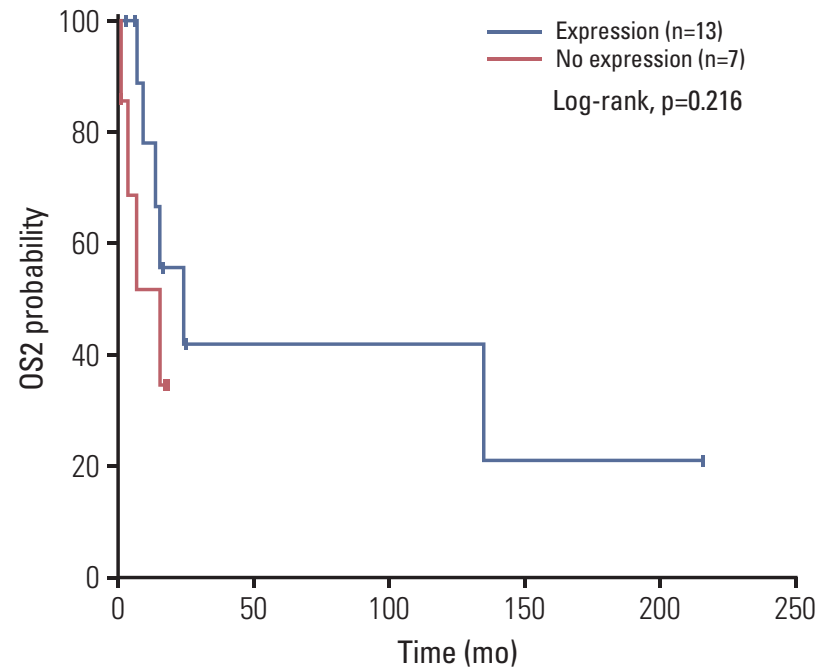

BAP1 IHC $(n=20)$

metastatic tumor

Median OS2 (mo)

$95 \% \mathrm{Cl}$

\begin{tabular}{lll} 
Expression $(\mathrm{n}=13)$ & 24.4 & $3.531-45.209$ \\
No expression $(\mathrm{n}=7)$ & 15.7 & $2.171-29.225$ \\
\hline
\end{tabular}

Fig. 1. Immunohistochemistry (IHC) analysis of primary uveal melanomas without (A) and with BRCA1 associated protein-1 (BAP1) expression (B) ( $\times 400)$. Analysis of recurrence-free survival (RFS, defined as the time from uveal melanoma diagnosis to recurrence) according to BAP1 expression (C). Analysis of overall survival from distant recurrence to death (OS2) according to BAP1 expression in metastatic tissues (D). CI, confidence interval; N/A, not available.

\section{Results}

1. Baseline characteristics of patients with primary and metastatic uveal melanoma

The baseline characteristics of 226 patients are provided in Table 1. The median age was 53.0 years (range, 18 to 71 years), and 109 patients (48.2\%) were male. The most common primary tumor location was the choroid $(\mathrm{n}=220,97.3 \%)$, and the most common histologic types were epithelioid $(\mathrm{n}=43,43.4 \%)$ and mixed cell $(\mathrm{n}=38,38.4 \%)$. The median tumor thickness was $6.2 \mathrm{~mm}$ (range, 1.10 to 757.9 ), and the median LBD was $10.2 \mathrm{~mm}$ (range, 1.1 to 30.9). According to the AJCC seventh edition prognostic staging definitions, 26 $(25.0 \%), 66(63.5 \%)$, and $12(11.5 \%)$ patients had stage I, II, 
Table 2. Univariate and multivariate analyses of risk factors affecting the recurrence of primary uveal melanoma

\begin{tabular}{|c|c|c|c|c|c|c|c|c|c|}
\hline \multirow{2}{*}{ Characteristic } & \multirow{2}{*}{ No. } & \multirow{2}{*}{$\begin{array}{l}\text { Median RFS } \\
(95 \% \mathrm{CI}, \mathrm{mo})\end{array}$} & \multirow{2}{*}{ p-value } & \multicolumn{3}{|c|}{ Univariate analysis } & \multicolumn{3}{|c|}{ Multivariate analysis } \\
\hline & & & & HR & $95 \% \mathrm{CI}$ & p-value & HR & $95 \% \mathrm{CI}$ & p-value \\
\hline \multicolumn{10}{|c|}{ Age, median (range, yr) } \\
\hline$<30$ & 17 & $163.0(\mathrm{~N} / \mathrm{A})$ & 0.929 & 1 & & & & & \\
\hline $30-60$ & 141 & Not reached & & 0.83 & $0.324-2.140$ & 0.703 & - & - & - \\
\hline$>60$ & 68 & Not reached & & 0.85 & $0.293-2.467$ & 0.765 & - & - & - \\
\hline \multicolumn{10}{|l|}{ Sex } \\
\hline Male & 109 & Not reached & 0.062 & 1.71 & $0.965-3.043$ & 0.066 & - & - & - \\
\hline Female & 117 & $163.0(\mathrm{~N} / \mathrm{A})$ & & 1 & & & & & \\
\hline \multicolumn{10}{|l|}{ Location } \\
\hline Choroid & 220 & $163.0(\mathrm{~N} / \mathrm{A})$ & 0.452 & 1 & & & & & \\
\hline Ciliary body & 6 & $88.0(\mathrm{~N} / \mathrm{A})$ & & 1.71 & $0.414-7.048$ & 0.459 & - & - & - \\
\hline \multicolumn{10}{|l|}{ Size } \\
\hline \multicolumn{10}{|c|}{$\begin{array}{l}\text { Largest basal diameter }(\mathrm{mm}) \\
(\mathrm{n}=223)\end{array}$} \\
\hline$<15$ & 201 & $163.0(\mathrm{~N} / \mathrm{A})$ & $<0.001$ & 1 & & & 1 & & \\
\hline$\geq 15$ & 22 & $25.0(0.00-61.18)$ & & 4.73 & $2.326-9.598<$ & $<0.001$ & 2.75 & $1.092-6.904$ & 0.032 \\
\hline \multicolumn{10}{|c|}{ Depth $(\mathrm{mm})(\mathrm{n}=224)$} \\
\hline$<10$ & 193 & $163.0(\mathrm{~N} / \mathrm{A})$ & $<0.001$ & 1 & & & 1 & & \\
\hline$\geq 10$ & 31 & 54.0 (N/A) & & 3.37 & $1.804-6.308<$ & $<0.001$ & 3.07 & $1.266-7.468$ & 0.013 \\
\hline \multicolumn{10}{|l|}{ Stage $(n=104)$} \\
\hline I & 26 & Not reached & 0.012 & 1 & & & & & \\
\hline II & 66 & $82.0(48.40-115.60$ & & 8.44 & $1.134-62.787$ & 0.037 & - & - & - \\
\hline III & 12 & $25.0(\mathrm{~N} / \mathrm{A})$ & & 15.04 & $1.752-129.043$ & 30.013 & - & - & - \\
\hline Total & 226 & $163.0(\mathrm{~N} / \mathrm{A})$ & & & & & & & \\
\hline
\end{tabular}

RFS, recurrence-free survival (time from the initial diagnosis of uveal melanoma to distant recurrence); $\mathrm{CI}$, confidence interval; HR, hazard ratio; N/A, not available.

and III disease, respectively. An IHC analysis identified 56 BAP1-positive samples $(81.2 \%)$ among 69 available primary tumor tissues.

Forty-nine patients $(21.6 \%)$ with primary uveal melanoma developed distant recurrences after local treatment. The baseline characteristics of these patients are also provided in Table 1. The most common location and histologic type of these recurrent uveal melanomas were choroid $(n=47,95.9 \%)$ and mixed cell $(\mathrm{n}=20,54.1 \%)$, respectively. The most common site of distant recurrence was the liver $(n=43,87.7 \%)$, followed by the bone $(n=11,22.4 \%)$ and lung $(n=3,12.2 \%)$.

\section{Treatment outcomes for primary and metastatic uveal melanoma}

Following a diagnosis of primary uveal melanoma, 160 patients $(70.8 \%)$ were treated with local radiotherapy, including ${ }^{106} \mathrm{Ru}$ brachytherapy combined with TTT and gammaknife radiotherapy; 66 patients $(29.2 \%)$ underwent excision, including primary enucleation, local resection, and TPPV. An analysis of RFS and OS1 according to treatment modality revealed a significantly longer in local radiotherapy than in excision (not reached vs. 82.0 months: 95\% confidence intervals [CI], not available [N/A] vs. 43.8-120.2; $\mathrm{p}<0.001$; not reached vs. 79.5 months: 95\% CI, N/A vs. 33.0-126.0; $\mathrm{p}<0.001)$.

Forty-nine patients with distant recurrences of uveal melanoma underwent metastasectomy $(n=7,14.3 \%)$, transarterial chemoembolization (TACE) or radiofrequency ablation (RFA) $(n=4,8.2 \%)$, local radiotherapy $(n=3,6.1 \%)$, or palliative systemic treatments, including chemotherapy and immunotherapy $(n=27,55.1 \%)$. Twelve patients who underwent metastasectomy, TACE/RFA, or radiotherapy subsequently received first-line palliative systemic treatment (S1 Fig.). The first-line systemic chemotherapy regimens included dacarbazine $(\mathrm{n}=27,77.1 \%)$, platinum-based agents $(n=7,20.0 \%)$, and topotecan $(n=1,2.9 \%)$. The first-line immunotherapies included interferon $(n=1,2.9 \%)$ and ipilimumab $(n=3,8.6 \%)$. Patients received a mean of 2.94 lines of palliative systemic treatment. 
A
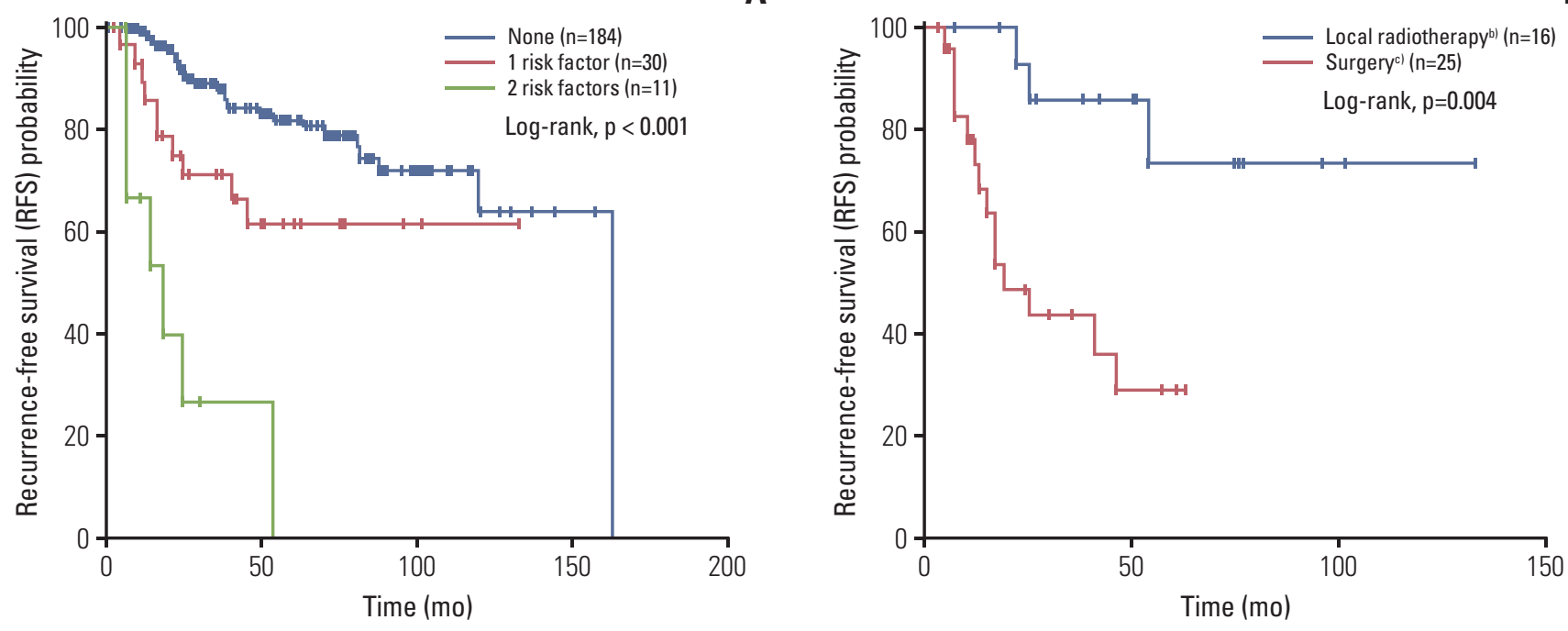

\begin{tabular}{lcc} 
Risk factor numbera) & Median RFS $(\mathrm{mo})$ & $95 \% \mathrm{Cl}$ \\
\hline None $(\mathrm{n}=184)$ & 163.0 & $\mathrm{~N} / \mathrm{A}$ \\
1 risk factor $(\mathrm{n}=30)$ & Not reached & N/A \\
2 risk factors $(\mathrm{n}=11)$ & 19.0 & $3.667-34.333$ \\
\hline
\end{tabular}

\begin{tabular}{lcc} 
Primary treatment & Median RFS (mo) & $95 \% \mathrm{Cl}$ \\
\hline Local radiotherapy) $(n=16)$ & Not reached & N/A \\
Surgery $^{\text {c) }}(n=25)$ & 19.0 & $8.402-29.598$ \\
\hline
\end{tabular}

Fig. 2. Analysis of recurrence-free survival (RFS, defined as the time from choroidal melanoma diagnosis to recurrence) according to risk factors for recurrence of primary uveal melanoma (A). (B) Subgroup analysis according to local treatment with $\geq 1$ risk factor. CI, confidence interval; N/A, not available. ${ }^{a}$ Includes tumor basal diameter $\geq 15 \mathrm{~mm}$, depth $\geq 10 \mathrm{~mm}$, b)Includes brachytherapy, gamma-knife radiotherapy, transpupillary thermotherapy, ${ }^{\mathrm{c}}$ Includes trans pars plana vitrectomy, enucleation, excision, exentration.

Among patients with distant recurrences of uveal melanoma, the DCR was analyzed according to treatment modality. Here, $71.4 \%(5 / 7)$ of patients with in metastasectomy followed by first-line systemic therapy, 33.3\% (9/29) of those receiving only first-line systemic therapy, and $0 \%$ of those receiving TACE or RFA or palliative radiotherapy followed by first-line systemic therapy achieved disease control (S2 Table). An analysis of median OS2 duration yielded values of 134.7 (95\% CI, 0.0 to 307.6), 8.0 (95\% CI, 6.8 to 9.2), 9.2 (95\% CI, N/A), 15.7 (95\% CI, 13.2 to 18.1), and 7.6 months (95\% CI, 0.0 to 25.2) for patients who underwent metastasectomy, TACE or RFA, palliative radiotherapy, only systemic treatments, and observation alone, respectively.

The median OS1 of overall 226 primary uveal melanoma patients was 151.6 months (95\% CI, 91.0 to 212.2) and the median OS2 of distant recurrent patients was 15.7 months (95\% CI, 13.1 to 18.2).

\section{Analysis of prognostic factors}

\section{1) BAP1 expression in primary and metastatic uveal mela- nomas}

In accordance with a previous study [8], we subjected 69 primary and 20 metastatic uveal melanoma tissues to BAP1 IHC (Fig. 1A and B). The loss of BAP1 expression was more frequent among metastatic tumor tissues than among primary tissues (13/69 [18.8\%] vs. 7/20 [35.0\%]) (Table 1). Patients with BAP1-negative primary tumor tissues had a longer median RFS when compared with their BAP1-positive counterparts, although this difference was not statistically significant (not reached vs. 82.0 months, $\mathrm{p}=0.268$ ) (Fig. 1C). However, patients with BAP1-negative metastatic tumor tissues had a shorter OS2 when compared to those with BAP1positive tissues; again, this difference was not statistically significant (15.7 months vs. 24.4 months, $\mathrm{p}=0.216$ ) (Fig. 1D). 


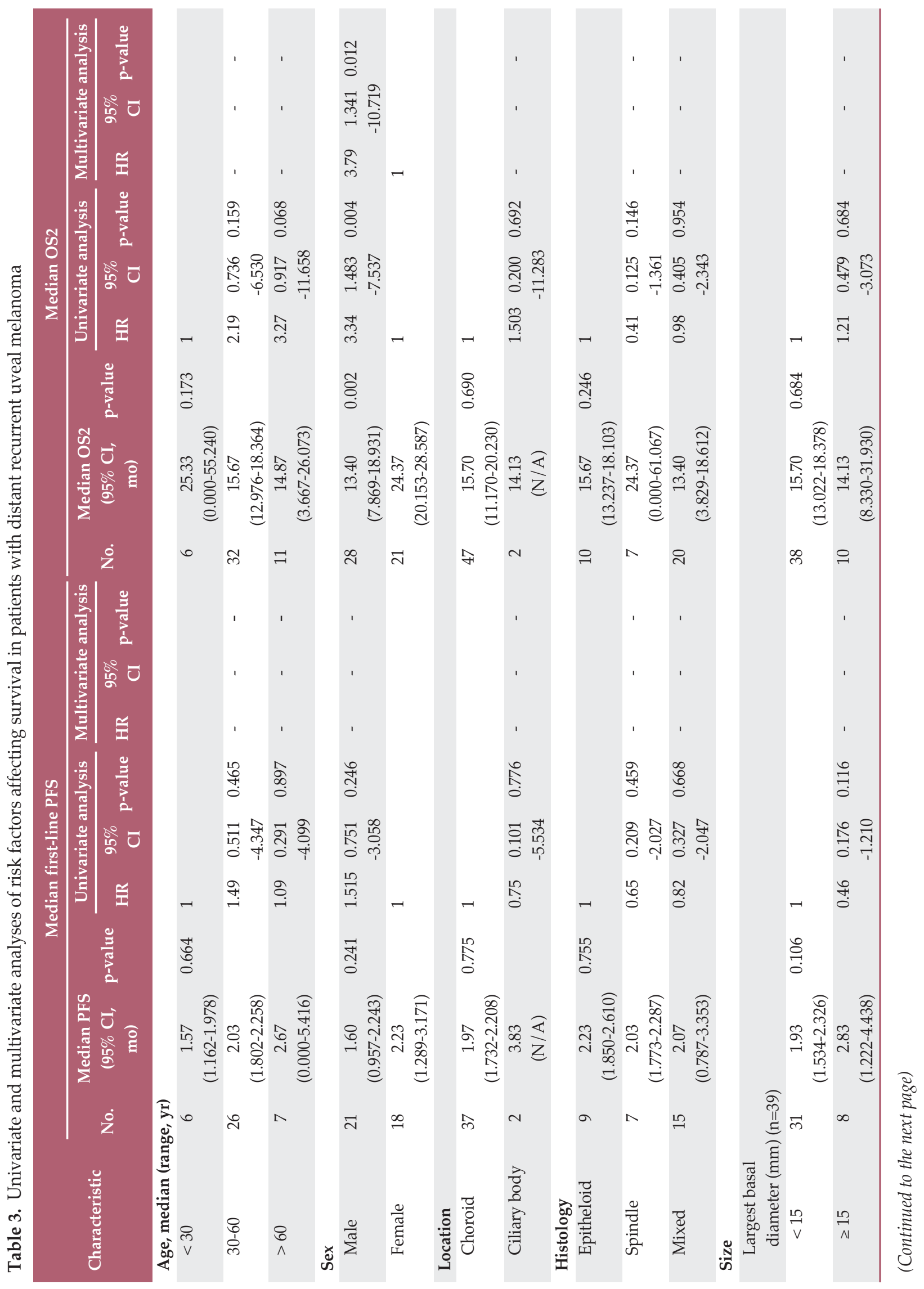




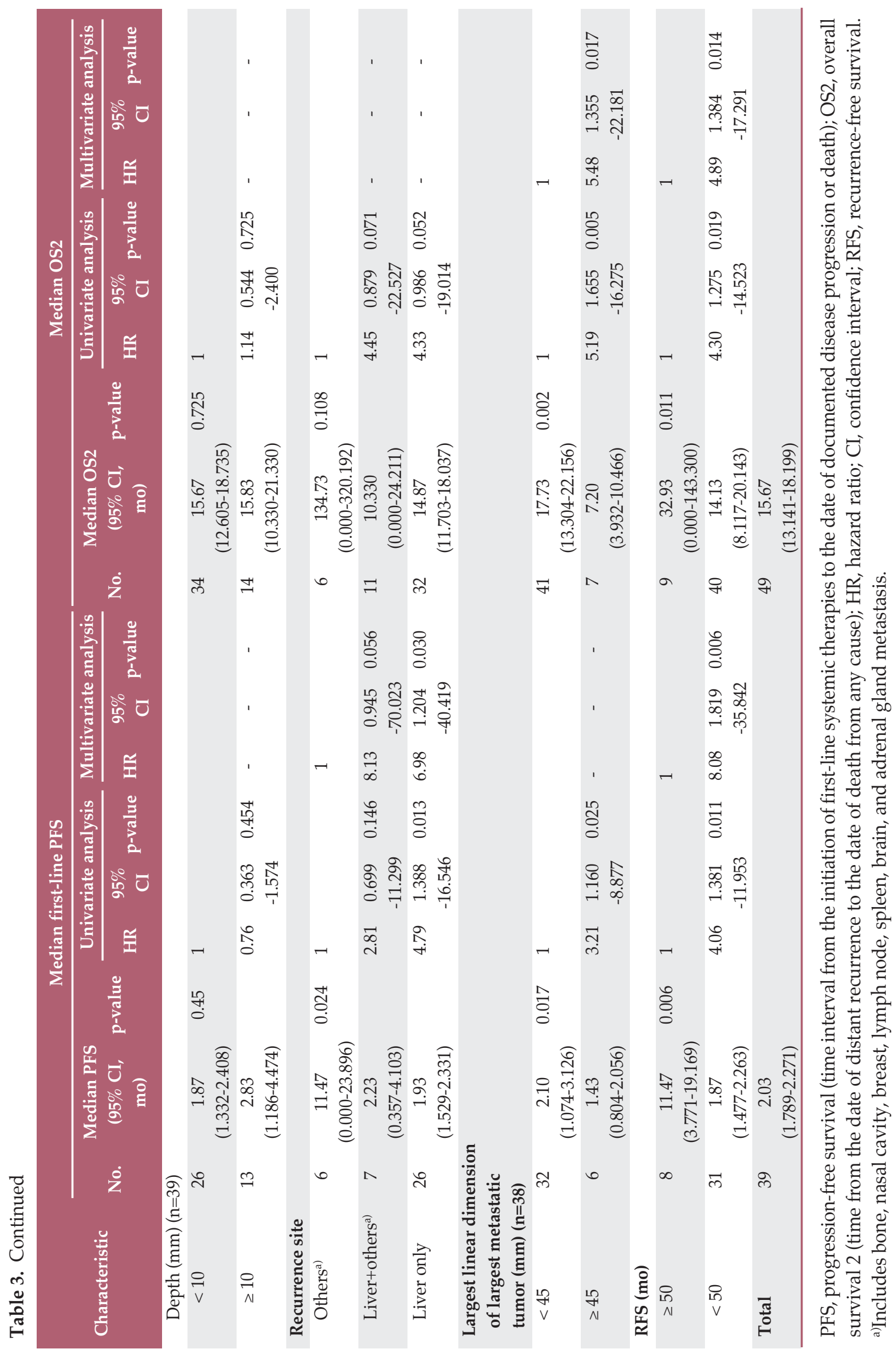


A
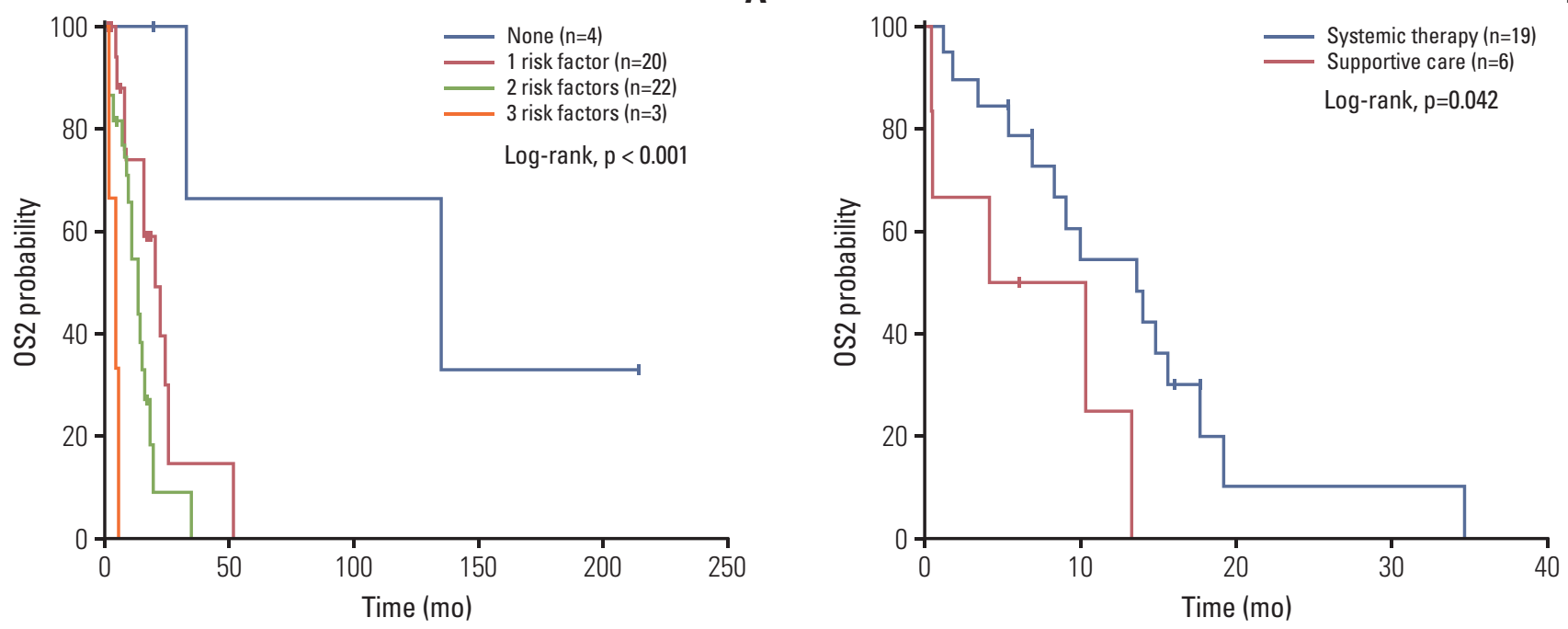

\begin{tabular}{|ccc}
\hline Risk factor $^{\mathrm{a})}$ & Median 0S2 (mo) & $95 \% \mathrm{Cl}$ \\
\hline None $(\mathrm{n}=4)$ & 134.7 & $0.000-297.644$ \\
\hline risk factor $(\mathrm{n}=20)$ & 19.8 & $10.429-29.111$ \\
\hline risk factors $(\mathrm{n}=22)$ & 13.4 & $8.415-18.385$ \\
3 risk factors $(\mathrm{n}=3)$ & 4.3 & $0.000-8.975$ \\
\hline
\end{tabular}

\begin{tabular}{lcc} 
Management & Median 0S2 (mo) & $95 \% \mathrm{Cl}$ \\
\hline Systemic therapy $(\mathrm{n}=19)$ & 13.7 & $7.247-20.153$ \\
Supportive care $(\mathrm{n}=6)$ & 4.3 & $0.000-13.584$ \\
\hline
\end{tabular}

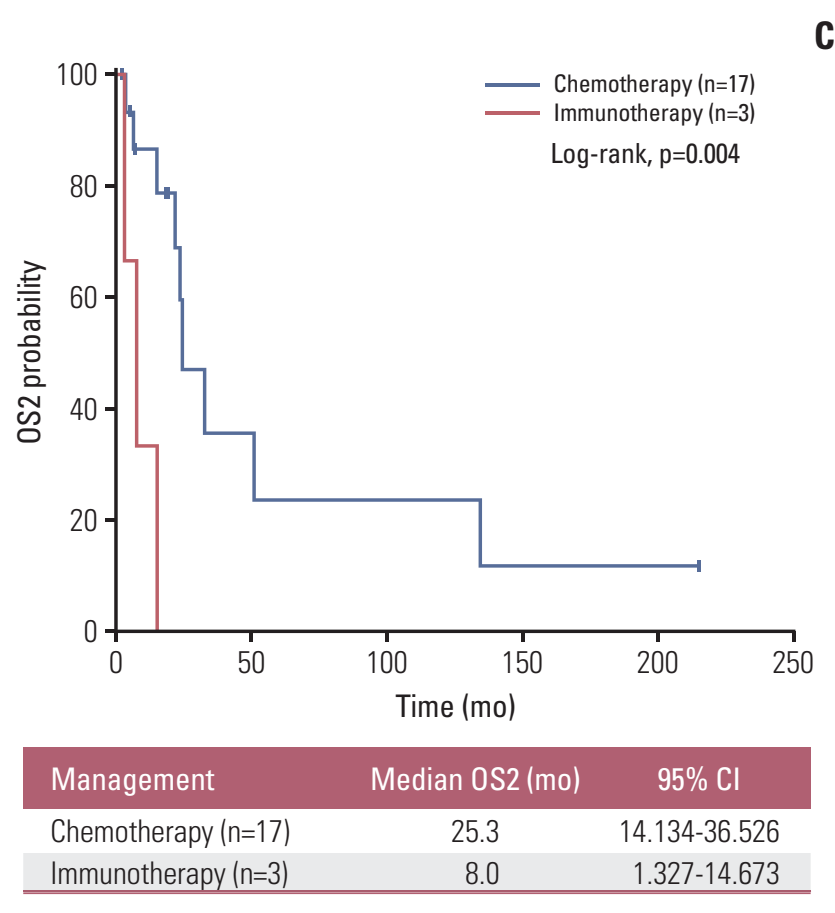

Fig. 3. Analysis of overall survival from distant recurrence to death (OS2) according to survival-related risk factors in patients with metastatic uveal melanoma (A). (B) Subgroup analysis of high-risk patients ( $\geq 2$ risk factors) according to systemic therapy or supportive care. (C) Subgroup analysis of low-risk patients ( $\leq 1$ risk factor) according to the type of systemic treatment. $\mathrm{CI}$, confidence interval. a) Includes male sex, largest linear dimension of largest metastatic tumor $\geq 45 \mathrm{~mm}$, recurrence-free survival $<50$ months. 


\section{2) Prognostic recurrent risk factors analysis of primary uveal melanoma}

We next analyzed prognostic factors for the recurrence of primary uveal melanomas following local treatments, as previously mentioned, to enhance risk-based patient stratification. In a univariate analysis, $\mathrm{LBD} \geq 15 \mathrm{~mm}(\mathrm{p}<0.001)$, depth $\geq 10 \mathrm{~mm}(\mathrm{p}<0.001)$, and stage II or III disease $(\mathrm{p}=0.037$ and $\mathrm{p}=0.013$, respectively) were found to have significant adverse effects on relapse (Table 2). A subsequent multivariate analysis included the clinical parameters of age, sex, location, tumor stage and size, and local treatment administration. A forward Cox regression model analysis identified the following significantly poor prognostic factors for relapse (Table 2): tumor basal diameter $\geq 15 \mathrm{~mm}$ (hazard ratio [HR], 2.75; 95\% CI, 1.09 to $6.90 ; \mathrm{p}=0.032)$, and depth $\geq 10 \mathrm{~mm}(\mathrm{HR}, 3.07 ; 95 \%$ CI, 1.27 to $7.47 ; \mathrm{p}=0.013$ ).

Next, 226 patients were divided into three subgroups based on multivariate results to identify those who would potentially benefit from primary local treatments: patients without any risk factors and those with 1-2 risk factors (Fig. 2A). Among the 41 patients with one or more risk factors, those who underwent local radiotherapy had a longer median RFS, compared to those who underwent local excision (not reached vs. 19.0 months; $95 \%$ CI, N/A vs. 8.4029.60; $\mathrm{p}=0.004$ ) (Fig. 2B).

\section{3) Analysis of prognostic and survival risk factors for recurrent uveal melanoma}

The univariate analysis identified the recurrence site (liver only, $\mathrm{p}=0.013$ ), a largest linear metastatic tumor dimension $\geq 45 \mathrm{~mm}(\mathrm{p}=0.025)$, and RFS $<50$ months ( $\mathrm{p}=0.011)$ as significantly poor prognostic factors for progression despite firstline systemic treatment (Table 3). In a multivariate analysis, the recurrence site (liver only; HR, 6.98; 95\% CI, 1.20 to 40.42; $\mathrm{p}=0.030$ ) and RFS < 50 months (HR, 8.08; 95\% CI, 1.82 to $35.84 ; \mathrm{p}=0.006$ ) remained significantly poor prognostic factors for PFS after first-line systemic therapy.

Regarding survival after recurrence, another univariate analysis identified male sex $(\mathrm{p}=0.004)$, a largest linear metastatic tumor dimension $\geq 45 \mathrm{~mm}(\mathrm{p}=0.005)$, and RFS $<50$ months $(\mathrm{p}=0.019)$ as factors with significant negative adverse effects on OS2 (Table 3). A forward Cox regression model analysis subsequently identified male sex (HR, 3.79; 95\% CI, 1.34 to 10.72; $\mathrm{p}=0.012$ ), a largest linear metastatic tumor dimension $\geq 45 \mathrm{~mm}$ (HR, 5.48; 95\% CI, 1.36 to 22.18; $\mathrm{p}=0.017$ ), and short RFS (HR, 4.89; 95\% CI, 1.38 to 17.29 ; $\mathrm{p}=0.014$ ) as significantly poor prognostic factors for survival after recurrence. Based on the multivariate analysis findings, 49 patients with distant recurrences of uveal melanoma were divided into four subgroups, and OS2 was found to decrease significantly as the number of risk factors increased (Fig. 3A). Among the 25 patients with two or more risk factors (highrisk group), 19 who received palliative first-line systemic treatment had a significantly longer OS2, compared to those who received supportive care only (13.7 months vs. 4.3 months, $\mathrm{p}=0.042$ ) (Fig. 3B). Seventeen of 20 patients with one or no risk factors (low-risk group) received first-line systemic therapy and in this group, first-line chemotherapy yielded a longer OS2 relative to immunotherapy (25.3 months vs. 8.0 months, $\mathrm{p}=0.004$ ) (Fig. 3C).

\section{Discussion}

Up to 50\% of patients with uveal melanoma will develop metastatic disease after primary local treatment [16]. Such cases are faced with a very poor prognosis and limited therapeutic options with low response rates. Several previous studies have reported the risk factors and characteristics of recurrent uveal melanoma [2-4,10,14,17-19].

In Korean patients, uveal melanomas tend to exhibit vertical growth, with relatively large apical heights and small LBDs [11]. In such cases, the initial radiation doses provided via brachytherapy at the time of diagnosis are insufficient to reach the basal level of the tumor, leading to frequent local and / or distant recurrences and an increased need for enucleation or systemic therapy. Regarding local treatment, our study found that 136 patients $(76.8 \%)$ in the non-recurrent group received local radiotherapy, while $25(51.0 \%)$ in the recurrent group underwent primary enucleation. Moreover, when compared with non-recurrent tumors, recurrent tumors had a significantly larger primary tumor basal size and significantly more advanced stage. The AJCC Ophthalmic Oncology Group reported that an increasing tumor size was consistent with an increased risk of metastasis [10], and a Taiwanese trial found associations of a larger tumor size and epithelioid or mixed cell type with distant metastasis [17]. Our findings were also supported by prior studies in which patients who underwent with primary enucleation had a higher recurrence rate than did those treated with irradiation [4].

As noted previously, Harbour et al. [7] reported an inactivating somatic mutation in BAP1, located on chromosome $3 p 21.1$, in $47 \%$ of all uveal melanomas; $96 \%$ of tumors harboring this mutation later metastasized. Koopmans et al. [8] confirmed that this somatic BAP1 mutation correlated strongly with BAP1 expression, and reported an eight-fold increase in the risk of metastasis among patients with BAP1negative or mutated BAP1-expressing uveal melanoma. In our study, however, we found that BAP1 expression in the 
primary tumor was associated with a shorter RFS, compared to a loss of BAP1 expression. Our multivariate analysis further identified two risk factors associated with distant recurrence: tumor basal diameter $\geq 15 \mathrm{~mm}$ and vertical depth $\geq 10$ $\mathrm{mm}$. We further found that in patients with at least one risk factor, local radiotherapy was more effective than local excision or enucleation in terms of RFS prolongation. Accordingly, we recommend that treatment strategies for primary uveal melanoma should be based on these risk factors.

Other investigators have described the patterns of metastatic spread in patients with uveal melanoma [20-23]. Although the liver and lung are the most frequent sites of metastasis, the sites and extent of dissemination vary among studies and reflect differences in study populations, designs, diagnostic tools, and data collection over time. Therefore, the Collaborative Ocular Melanoma Study (COMS) protocol specifies an annual follow-up of patients, including a routine medical examination, liver function studies, and chest $\mathrm{X}$-ray examination [24]. In addition, Hicks et al. [25] recommended routine liver ultrasonography follow-ups after determining the poor sensitivity of a single liver function test for metastatic disease. In our study, the first distant metastatic site in 38 patients $(77.6 \%)$ was a single organ. Although the liver was the first metastatic site in $81.6 \%$ of patients with single-organ involvement and $63.3 \%$ of all patients, nearly $40 \%$ of all patients presented with non-liver sites of first metastasis (mainly bone). This finding suggests that liver-only screening procedures would miss a substantial proportion of patients. Therefore, guidelines for periodic systemic surveillance should be established for patients in Asia, and particularly those in Korea who have undergone definitive local treatment for primary uveal melanoma.

Although metastatic uveal melanoma is considered a substantial problem, relatively few studies have addressed factors associated with survival. The median survival times of the two largest unselected groups of patients with metastatic uveal melanoma in the COMS and Harvard study were 3.6 and 3.7 months $[26,27]$. The COMS study reported 1-year and 2-year survival rates of only $19 \%$ and $8 \%$, respectively [27]. In our study, we observed a median OS2 of 15.7 months, in contrast to previous studies. We might attribute this discrepancy to the fact that all patients in our study population received local ocular treatment at the time of primary uveal melanoma diagnosis and remained disease-free for a median of 38.1 months. In contrast to previous studies that included only patients initially diagnosed with stage IV disease, we might have observed a lower level of aggressiveness because the recurrences occurred substantially later after local treatment. Additionally, our study included patients who received palliative treatments after metastasectomy from the liver, adrenal gland, or breast mass or after radiotherapy. In a more accurate comparison of patients who underwent metastasectomy with those who received only systemic firstline treatment, the former had a significantly longer survival duration. In addition, patients who received only supportive care had a significantly longer median OS2 duration, compared to those than those who received TACE, RFA, or radiotherapy. Therefore, we would expect the surgical resection of metastatic lesions to yield survival benefits.

In various studies of different groups of patients with metastatic uveal melanoma, the investigators identified multiple predictors of survival duration after the diagnosis of metastasis. The identified poor prognostic factors included patient age, male sex, symptomatic tumor, poor performance status, short metastasis-free interval, anatomic site with metastatic involvement (i.e., hepatic involvement), large number of metastatic sites, and metastatic lesion size $[4,28]$. In our study, a multivariate analysis identified male sex, a largest linear metastatic tumor diameter $\geq 45 \mathrm{~mm}$, and a short RFS as prognostic factors associated with OS2. As previously observed, high-risk patients ( $\geq 2$ risk factors) who received systemic first-line therapy after distant recurrence had a longer survival duration, compared to those treated with only supportive care. Therefore, high-risk patients should be identified and treated with systemic therapies, as this would be expected to improve survival rates.

Medical oncologists are required to select from among chemotherapy, immunotherapy, or targeted agents when determining the systemic treatment most likely to improve the survival of a patient with metastatic uveal melanoma. However, no effective systemic treatment method has yet been identified, and no reports of non-randomized phase III clinical trials of any alternative or other specific treatments have been published. We note that in our study, the first-line treatments provided for metastatic uveal melanoma varied considerably by subgroup and included surgery, TACE, and/or radiotherapy. The retrospective design of our study limited our analysis of the effect of systemic treatment after relapse. However, given the heterogeneous nature of treatments received by patients in this study, we should be careful when interpreting the efficacies of these first-line systemic treatments. We further note that we have not identified an ideal first-line systemic therapeutic strategy that significantly improved survival, although among patients who received only palliative systemic treatment without surgery, TACE, or radiation for metastatic lesions, those treated with chemotherapy had a significantly longer median OS2, compared to those receiving mmunotherapy (S3 Table). A similar OS2 outcome was also observed among low-risk patients. Among patients receiving only first-line chemotherapy $(n=26)$, a platinum-based regimen was associated with a longer PFS relative to dacarbazine or topotecan. However, dacarbazine seemed to elicit a more durable response and longer OS2 when compared with platinum-based and topotecan, 
although these differences were not statistically significant (S4 Fig.).

Our study had the following strengths. In our cohort, approximately $40 \%$ of patients presented with a non-hepatic first metastasis, which suggests that liver-only screening strategies are likely to fail. Accordingly, bone and chest examinations should also be included in screening protocols, as the early detection of recurrent metastatic lesions will increase the likelihood of surgical resection. Notably, metastasectomy correlated with improved survival in our dataset. Furthermore, relevant studies specific to Asian populations are limited. Our study, which assessed real efficacy in a population of Korean patients, therefore provides important information. Moreover, clinical data were used to score the factors related to overall survival after recurrence and to suggest treatment strategies.

However, this study also had two main limitations. First, we did not conduct gene expression profiling or a full analysis of mutations in genes such as GNAQ, GNA11, or BAP1, which are known poor prognosticators in uveal melanoma. Instead, we confirmed BAP1 expression in primary and metastatic tissues using IHC. Our finding of a high risk of recurrence among patients with BAP1-positive primary tumor tissues contrasted with a previous report [8]. On the other hand, van Essen et al. [29] reported that low RNA levels of BAP1 and negative IHC for BAP1 were predictive of death due to metastasis of uveal melanoma. We also observed an association of the loss of BAP1 expression in metastatic tumors with poor survival in patients with recurrent uveal melanoma patients. These inter-study discrepancies suggest that we should validate our findings using a BAP1 mutation analysis. Second, this study was performed at a single center and featured a retrospective design, and we were unable to compare the effects associated with immunotherapy, chemotherapy, and targeted agent therapy because of the small number of patients with distant recurrences. Therefore, multicenter prospective studies are needed to further estimate the effects of treatment on survival in patients with recurrent uveal melanoma.

In conclusion, the present study conducted a multivariate analysis of clinical and histologic data to identify risk factors related to recurrence in patients with primary uveal melanoma and survival in those with distant recurrent uveal melanoma. Our findings suggest that patients with primary uveal melanoma and one or more risk factors would more strongly benefit from local radiotherapy (vs. local excision or primary enucleation) for recurrence prevention. We also found that for "high risk" patients with recurrent uveal melanoma, more aggressive systemic first-line chemotherapeutic regimens and surgical treatment would likely improve survival. Finally, a multidisciplinary approach combining ophthalmology, radiation oncology, general surgery, and medical oncology should help to improve overall survival in patients with primary and recurrent uveal melanoma.

\section{Electronic Supplementary Material}

Supplementary materials are available at Cancer Research and Treatment website (http:// www.e-crt.org).

\section{Conflicts of Interest}

Conflict of interest relevant to this article was not reported.

\section{Acknowledgments}

This research was supported by a faculty research grant from the Yonsei University College of Medicine for 2014 (6-2014-0166).

\section{References}

1. Augsburger JJ, Schneider S, Freire J, Brady LW. Survival following enucleation versus plaque radiotherapy in statistically matched subgroups of patients with choroidal melanomas: results in patients treated between 1980 and 1987. Graefes Arch Clin Exp Ophthalmol. 1999;237:558-67.

2. Kujala E, Makitie T, Kivela T. Very long-term prognosis of patients with malignant uveal melanoma. Invest Ophthalmol Vis Sci. 2003;44:4651-9.

3. Singh AD, Turell ME, Topham AK. Uveal melanoma: trends in incidence, treatment, and survival. Ophthalmology. 2011; 118:1881-5.
4. Koutsandrea C, Moschos MM, Dimissianos M, Georgopoulos G, Ladas I, Apostolopoulos M. Metastasis rates and sites after treatment for choroidal melanoma by proton beam irradiation or by enucleation. Clin Ophthalmol. 2008;2:989-95.

5. Yamazaki N, Takenouchi T, Fujimoto M, Ihn H, Uchi H, Inozume $\mathrm{T}$, et al. Phase $1 \mathrm{~b}$ study of pembrolizumab (MK-3475; anti-PD-1 monoclonal antibody) in Japanese patients with advanced melanoma (KEYNOTE-041). Cancer Chemother Pharmacol. 2017;79:651-60.

6. Kottschade LA, McWilliams RR, Markovic SN, Block MS, Villasboas Bisneto J, Pham AQ, et al. The use of pembrolizumab 
for the treatment of metastatic uveal melanoma. Melanoma Res. 2016;26:300-3.

7. Harbour JW, Onken MD, Roberson ED, Duan S, Cao L, Worley LA, et al. Frequent mutation of BAP1 in metastasizing uveal melanomas. Science. 2010;330:1410-3.

8. Koopmans AE, Verdijk RM, Brouwer RW, van den Bosch TP, van den Berg MM, Vaarwater J, et al. Clinical significance of immunohistochemistry for detection of BAP1 mutations in uveal melanoma. Mod Pathol. 2014;27:1321-30.

9. Khattak MA, Fisher R, Hughes P, Gore M, Larkin J. Ipilimumab activity in advanced uveal melanoma. Melanoma Res. 2013;23:79-81.

10. AJCC Ophthalmic Oncology Task Force. International Validation of the American Joint Committee on Cancer's 7th edition classification of uveal melanoma. JAMA Ophthalmol. 2015; 133:376-83.

11. Kwon HJ, Ko JS, Kim M, Lee CS, Lee SC. Prognosis of choroidal melanoma and the result of ruthenium brachytherapy combined with transpupillary thermotherapy in Korean patients. Br J Ophthalmol. 2013;97:653-8.

12. Lee JH, Lee SC, Cho A, Keum KC, Suh YG, Lee CS. Association between choroidal thickness and metabolic activity on positron emission tomography in eyes with choroidal melanoma. Am J Ophthalmol. 2015;160:1111-5.e2.

13. Damato BE, Foulds WS. Surgical resection of choroidal melanoma. In: Schachat AP, Ryan SK, editors. Retina. St. Louis, MO: Mosby; 2001. p. 762-72.

14. Foulds WS, Damato BE, Burton RL. Local resection versus enucleation in the management of choroidal melanoma. Eye (Lond). 1987;1(Pt 6):676-9.

15. Damato B, Groenewald C, McGalliard J, Wong D. Endoresection of choroidal melanoma. Br J Ophthalmol. 1998;82:213-8.

16. Buder K, Gesierich A, Gelbrich G, Goebeler M. Systemic treatment of metastatic uveal melanoma: review of literature and future perspectives. Cancer Med. 2013;2:674-86.

17. Liu YC, Tsai CC, Lee FL, Lee SM, Kao SC. Mortality from uveal melanoma treated by enucleation: a 16-year survey in Taiwan. Acta Ophthalmol. 2013;91:e583-4.

18. Murali R, Wiesner T, Scolyer RA. Tumours associated with
BAP1 mutations. Pathology. 2013;45:116-26.

19. Rietschel P, Panageas KS, Hanlon C, Patel A, Abramson DH, Chapman PB. Variates of survival in metastatic uveal melanoma. J Clin Oncol. 2005;23:8076-80.

20. Collaborative Ocular Melanoma Study Group. Assessment of metastatic disease status at death in 435 patients with large choroidal melanoma in the Collaborative Ocular Melanoma Study (COMS): COMS report no. 15. Arch Ophthalmol. 2001; 119:670-6.

21. Kath R, Hayungs J, Bornfeld N, Sauerwein W, Hoffken K, Seeber S. Prognosis and treatment of disseminated uveal melanoma. Cancer. 1993;72:2219-23.

22. Bedikian AY, Legha SS, Mavligit G, Carrasco CH, Khorana S, Plager $C$, et al. Treatment of uveal melanoma metastatic to the liver: a review of the M. D. Anderson Cancer Center experience and prognostic factors. Cancer. 1995;76:1665-70.

23. Pyrhonen S. The treatment of metastatic uveal melanoma. Eur J Cancer. 1998;34 Suppl 3:S27-30.

24. Collaborative Ocular Melanoma Study Group. COMS Manual of Procedures (1999). NTIS accession No. PB95-179693. Springfield: National Technical Information Service; 1999.

25. Hicks C, Foss AJ, Hungerford JL. Predictive power of screening tests for metastasis in uveal melanoma. Eye (Lond). 1998;12(Pt 6):945-8.

26. Gragoudas ES, Egan KM, Seddon JM, Glynn RJ, Walsh SM, Finn SM, et al. Survival of patients with metastases from uveal melanoma. Ophthalmology. 1991;98:383-9.

27. Diener-West M, Reynolds SM, Agugliaro DJ, Caldwell R, Cumming K, Earle JD, et al. Screening for metastasis from choroidal melanoma: the Collaborative Ocular Melanoma Study Group Report 23. J Clin Oncol. 2004;22:2438-44.

28. Augsburger JJ, Correa ZM, Shaikh AH. Effectiveness of treatments for metastatic uveal melanoma. Am J Ophthalmol. 2009;148:119-27.

29. van Essen TH, van Pelt SI, Versluis M, Bronkhorst IH, van Duinen SG, Marinkovic M, et al. Prognostic parameters in uveal melanoma and their association with BAP1 expression. Br J Ophthalmol. 2014;98:1738-43. 